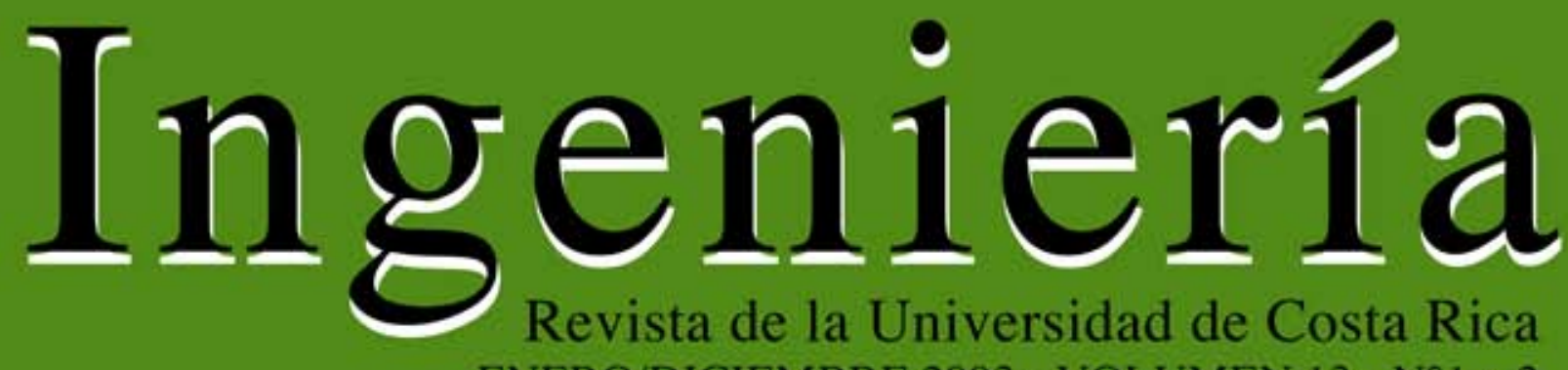
ENERO/DICIEMBRE 2003 - VOLUMEN 13 - N¹ y 2

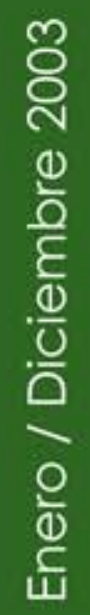

$\frac{m}{5}$
$\frac{c}{d}$
$\frac{5}{5}$
$\frac{\partial}{\circ}$

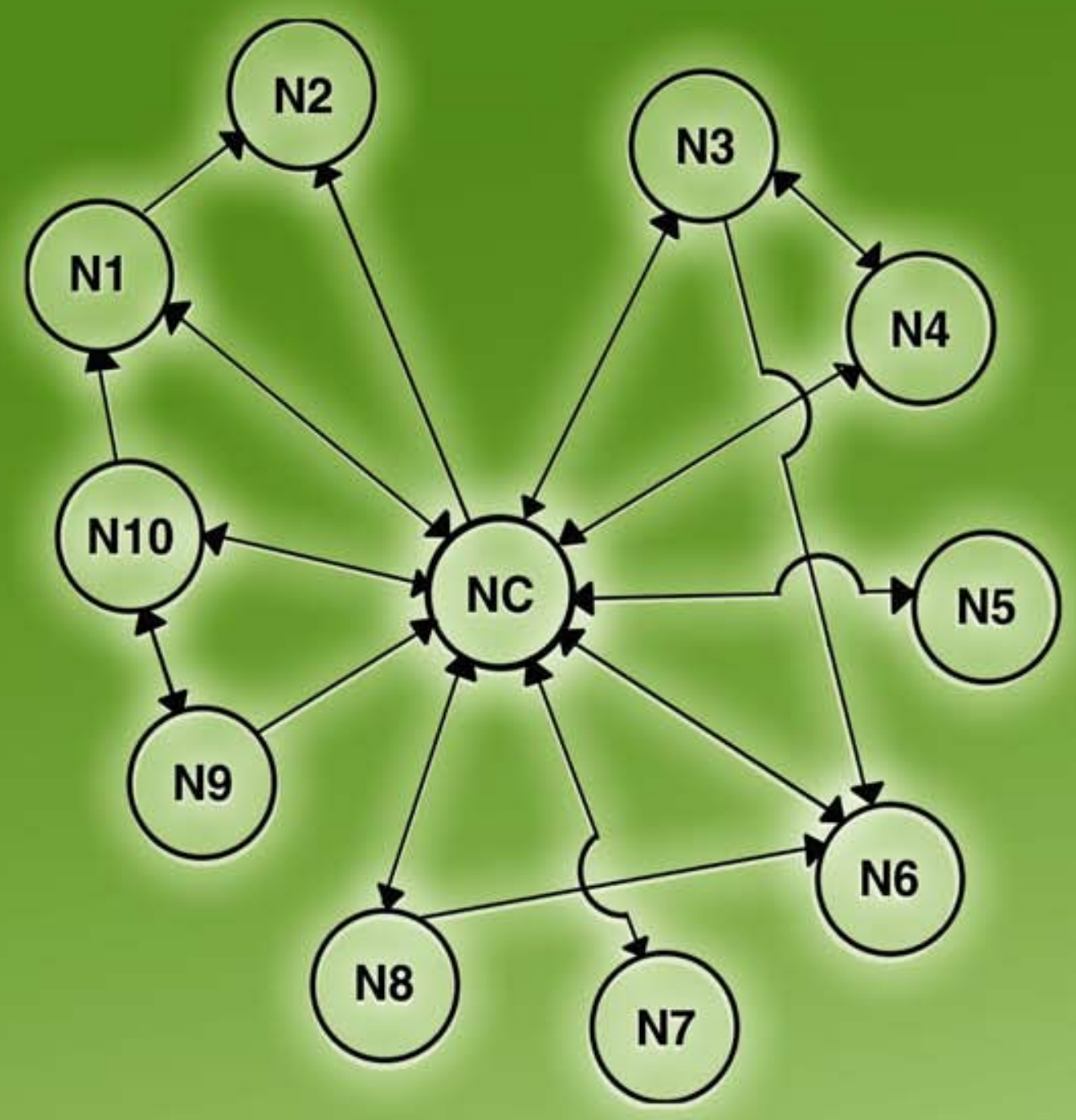

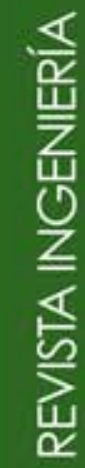




\title{
SINTONIZACIÓN DE CONTROLADORES PI Y PID UTILIZANDO LOS CRITERIOS INTEGRALES IAE E ITAE
}

Orlando Arrieta Orozco

Víctor M. Alfaro Ruiz.

\begin{abstract}
Resumen
Se plantea en general el problema de la determinación de los parámetros óptimos de controladores bajo los criterios integrales y se describen las características de los métodos de sintonización de controladores de López, Rovira, y de Kaya y Sheib.

Se presentan nuevas ecuaciones para el cálculo de los parámetros óptimos para controladores PI y PID bajo los criterios integrales IAE e ITAE.

Mediante pruebas comparativas por simulación, se muestran las ventajas obtenidas con las nuevas ecuaciones presentadas.
\end{abstract}

Palabras clave: controladores PID, sintonización, criterios integrales (IAE, ITAE)

\begin{abstract}
The general problem of finding the controller optimal parameters using integral performance criteria is presented. The tuning method developed by López, Rovira, and Kaya and Sheib are described.

A new set of equations to obtain the optimal parameters of a PI and PID controllers under the IAE and ITAE performance criteria are presented.

Using simulation comparisons, the advantages of the new tuning relations are demonstrated.
\end{abstract}

Keywords: PID controllers, tuning rules, integral criteria (IAE, ITAE)

\section{INTRODUCCIÓN}

La operación satisfactoria de un lazo de control realimentado como el mostrado en la Figura 1 , requiere de una adecuada sintonización del controlador, procedimiento para el cual es necesario determinar primeramente el tipo de controlador que se empleará, cual será el funcionamiento requerido del sistema, servomecanismo o regulador, y cual criterio se empleará para medir su desempeño.

El controlador más empleado en la industria es el PID (Proporcional-Integral-Derivativo), el cual en muchos de los casos opera simplemente como un controlador PI debido a las dificultadas encontradas normalmente en la utilización del modo derivativo en aplicaciones donde las señales pueden contener ruido de medición.
Como se aprecia en la Figura 1, existen dos entradas al sistema de control, el valor deseado $r(t)$ y la perturbación $z(t)$. Cuando se desea que la variable controlada $y(t)$ siga a un valor deseado cambiando con el tiempo, se dice que el sistema de control debe operar como servomecanismo, y cuando lo requerido es mantener la variable controlada en su valor deseado ante cambios en las perturbaciones, que debe operar como regulador.

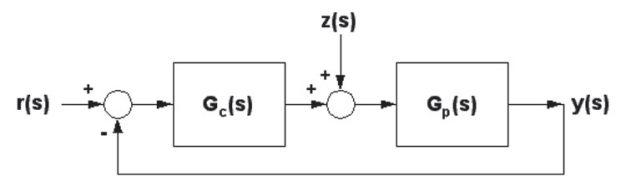

Figura 1. Sistema de control realimentado. 
El criterio empleado para la sintonización del controlador está relacionado directamente con el desempeño esperado del lazo de control y puede basarse en características deseadas de la respuesta en el tiempo o la frecuencia. Por ejemplo el primer método de sintonización de controladores, desarrollado por (Ziegler y Nichol, 1942), se basó en lo que denominaron "decaimiento de 1/4", esto es que la magnitud del segundo máximo de la señal de error ante un cambio en la perturbación, fuera la cuarta parte del valor del primero.

El decaimiento de $1 / 4$ fue seleccionado como un compromiso entre error máximo y velocidad de respuesta y las ecuaciones derivadas para la sintonización de los controladores fueron el resultado de múltiples pruebas experimentales con una planta piloto.

\subsection{Criterios integrales}

La búsqueda de una forma para cuantificar el comportamiento de los lazos de control llevó al establecimiento de índices de desempeño basados en la señal de error e $(t)$, la cual es la diferencia entre el valor deseado de la variable controlada y su valor real. De estos los más conocidos son los llamados criterios integrales definidos como:

- Integral del error absoluto

$$
I A E=\int_{0}^{\infty}|e(t)| d t
$$

- Integral del tiempo por el error absoluto

$$
\text { ITAE }=\int_{0}^{\infty} t|e(t)| d t
$$

- Integral del error cuadrático

$$
I S E=\int_{0}^{\infty} e(t)^{2} d t
$$

- Integral del tiempo por el error cuadrático

$$
\operatorname{ITSE}=\int_{0}^{\infty} t e(t)^{2} d t
$$

en donde el error está dado por

$$
e(t)=r(t)-y(t)
$$

Entonces para una planta dada, el objetivo es determinar los parámetros del controlador que minimizan la función de costo escogida, por lo que estos parámetros son óptimos bajo el criterio de desempeño establecido.

\subsection{Métodos de sintonización de controladores}

(Willis, 1962a) y (Willis, 1962b) fue uno de los primeros en utilizar los criterios integrales para estudiar el efecto de los parámetros del controlador sobre el valor de estos índices, mostrando sus conclusiones en forma gráfica.

Poco después y como parte de un proyecto de investigación comandado por los profesores C.L. Smith y P.W. Murrill, se obtuvieron en la Universidad del Estado de Louisiana (EUA) los dos métodos de sintonización de controladores basados en los criterios integrales más conocidos.

Estos métodos requieren de la identificación de un modelo de primer orden más tiempo muerto para la planta dado por

$$
G_{p}(s)=\frac{k_{p} e^{-t_{m} s}}{\tau s+1}
$$

en donde $\mathrm{k}_{\mathrm{p}}, \mathrm{t}$ y $\mathrm{t}_{\mathrm{m}}$ son la ganancia, la constante tiempo y el tiempo muerto del modelo.

(López et al, 1967) estudiaron los controladores $P$, PI y PID-Ideal con los criterios integrales IAE, ITAE e ISE para sistemas operando como reguladores y determinaron ecuaciones de sintonización válidas para valores de $t_{m} / \quad$ 1,0.

Por su parte (Rovira et al,1969) lo hicieron para controladores PI y PID-Ideal operando como servomecanismo, con los criterios IAE e ITAE y determinaron ecuaciones de sintonización válidas también para valores de $t_{m} / \quad 1,0$. 
Como se ha indicado anteriormente, las ecuaciones de López y las de Rovira se pueden aplicar directamente para el cálculo de los parámetros de un controlador PID-Ideal cuya ecuación es

$$
\begin{aligned}
& G_{C}(s)=K_{C}\left(1+\frac{1}{T_{i} s}+\frac{T_{d} s}{\alpha T_{d} s+1}\right) \\
& \quad=0,1 .
\end{aligned}
$$

Posteriormente (Kaya y Sheib, 1988) realizaron nuevamente el estudio para determinar los parámetros de los controladores optimizados con los criterios IAE, ITAE e ISE para valores de $t_{m} / 1,0$. Los controladores utilizados por Kaya y Sheib en su estudio fueron el PID-Serie, una variante del PID-Paralelo y el PID-Industrial. Las características de estos controladores y la conversión de sus parámetros a los de un PIDIdeal son presentadas por (Alfaro, 1993).

Otro método de sintonización basado en criterios integrales es el (Sung et al, 1996), pero debido a que requiere identificar un modelo de segundo orden más tiempo muerto para la planta, su utilización no es tan difundida como la de los otros métodos mencionados.

\section{NUEVO MÉTODO DE SINTONIZACIÓN}

Como parte de un estudio sobre el desempeño de los controladores $P I$ y $P I D$ sintonizados con criterios integrales, (Arrieta, 2003) determinó que era posible obtener mejores índices de desempeño $I A E$ e ITAE, especialmente en sistemas de control PID operando como servomecanismo.

A continuación se describe el procedimiento empleado para la determinación de los parámetros óptimos y el establecimiento de estas nuevas ecuaciones de sintonización; se presentan ejemplos de su utilización y se compara su desempeño con el de los otros métodos ya citados.

\subsection{Planteamiento del problema}

Se deben encontrar los parámetros de un controlador $P I\left(K_{c}, T_{i}\right)$ y de un controlador PID-Ideal $\left(K_{c}, T_{i}, T_{d}\right)$ que minimicen los criterios integrales IAE e ITAE operando como servomecanismo y como regulador para plantas de primer orden más tiempo muerto como la dada por (6).

La optimización se realizó simulando el lazo de control con el programa VisSim $3.0^{10}$ con una planta con ganancia $k_{p}=1,0$, constante de tiempo $=1,0 \mathrm{y}$ valores de tiempo muerto $t_{m}=0,1 \quad 0,4$ 0,8 y 1,2 , empleando el método de Euler para un intervalo de tiempo de 0 a $20 \mathrm{~s}$ con un paso de integración de $0,001 \mathrm{~s}$.

\subsection{Ecuaciones de sintonización}

Con base en los parámetros óptimos obtenidos y utilizando la función para ajuste de curvas por mínimos cuadrados de MATLAB (Coleman et al, 1999, se determinaron las siguientes ecuaciones de sintonización:

$K_{C} k_{p}=a+b\left(\frac{t_{m}}{\tau}\right)^{c}$

$$
\frac{T_{i}}{\tau}=d+e\left(\frac{t_{m}}{\tau}\right)^{f}
$$

$$
\frac{T_{d}}{\tau}=g+h\left(\frac{t_{m}}{\tau}\right)^{i}
$$

en donde los valores de las constantes $a$ hasta $i$ dependen del tipo de controlador, el funcionamiento del lazo y el criterio optimizado.

En el Cuadro 1 se listan las constantes de las ecuaciones (8) a (10) para reguladores y en el Cuadro 2 las correspondientes a servomecanismos. 
Cuadro 1. Reguladores - Constantes de las ecuaciones de sintonización

\begin{tabular}{|c|c|c|c|c|c|c|c|c|c|c|}
\hline Criterio & Controlador & $\mathbf{a}$ & b & c & d & e & f & g & $\mathbf{h}$ & $\mathbf{i}$ \\
\hline \multirow[t]{2}{*}{ IAE } & PI & 0,4485 & 0,6494 & $-1,1251$ & $-0,2551$ & 1,8205 & 0,4749 & - & - & - \\
\hline & PID & 0,1050 & 1,2432 & $-0,9946$ & $-0,2512$ & 1,3581 & 0,4796 & $-0,0003$ & 0,3838 & 0,9479 \\
\hline \multirow[t]{2}{*}{ ITAE } & PI & 0,2607 & 0,6470 & $-1,1055$ & $-1,5926$ & 2,9191 & 0,1789 & - & - & - \\
\hline & PID & 0,1230 & 1,1891 & $-1,0191$ & $-0,3173$ & 1,4489 & 0,4440 & $-0,0053$ & 0,3695 & 0,9286 \\
\hline
\end{tabular}

Cuadro 2. Servomecanismos - Constantes de las ecuaciones de sintonización

\begin{tabular}{ccccccccccc} 
Criterio & Controlador & $\mathbf{a}$ & $\mathbf{b}$ & $\mathbf{c}$ & $\mathbf{d}$ & $\mathbf{e}$ & $\mathbf{f}$ & $\mathbf{g}$ & $\mathbf{h}$ & $\mathbf{i}$ \\
IAE & PI & 0,2438 & 0,5305 & $-1,0299$ & 0,9377 & 0,4337 & 0,8714 & - & - & - \\
& PID & 0,2268 & 0,8051 & $-0,9597$ & 1,0068 & 0,3658 & 1,0092 & $-0,0146$ & 0,3500 & 0,8100 \\
\multirow{2}{*}{ ITAE } & PI & 0,1140 & 0,5131 & $-1,0382$ & 0,9953 & 0,2073 & 1,5246 & - & - & - \\
& PID & 0,1749 & 0,8355 & $-0,9462$ & 0,9581 & 0,3987 & 0,6884 & $-0,0169$ & 0,3126 & 0,7417
\end{tabular}

\subsection{Comparación con otros métodos}

En los Cuadros 3 y 4 se muestran los valores de los índices de desempeño integrales IAE e ITAE obtenidos con los diferentes métodos de sintonización para controladores $P I$ y en los Cuadros 5 y 6 para los controladores PID. La evaluación de los índices integrales se realizó para un intervalo de tiempo de 0 a $20 \mathrm{~s}$, ante cambios escalón unitario en el valor deseado y en la perturbación.

Para el caso de reguladores y servomecanismos PI, el método de Arrieta muestra una ligera ventaja sobre el método de López y el de Rovira para valores altos de la relación $t_{m} /$.

Algo similar ocurre con los controladores PID como regulador en donde el nuevo método supera al de López y al de Kaya y Sheib en forma apreciable para valores altos de la relación $t_{m} /$. Se puede notar que en este caso el método de López es mejor que el de Kaya y Sheib.

La diferencia más marcada y en donde el nuevo método de sintonización provee resultados mucho mejores, es para el caso de controladores PID operando como servomecanismo, donde el método de Kaya y Sheib supera al de Rovira excepto en un caso y el nuevo método es mejor que ambos para todas las relaciones de $t_{m} /$ estudiadas.

\subsection{Ejemplo}

Las comparaciones hechas en el punto anterior se basaron en el control de una planta de primer orden más tiempo muerto, esto es, como si el modelo utilizado para la sintonización fuera idéntico a la planta controlada.

En la práctica los procesos industriales son de orden alto los cuales deben aproximarse por un modelo de primer orden más tiempo muerto para aplicar las ecuaciones de sintonización.

Para comparar el desempeño de los diferentes métodos presentados, se empleó una de las plantas de prueba sugeridas por (Aström y Hägglund, 2000), dada por la función de transferencia 
$G_{p}(s)=\frac{1}{(s+1)(\lambda s+1)\left(\lambda^{2} s+1\right)\left(\lambda^{3} s+1\right)}$

con valores $=0,1 \quad 0,2 \quad 0,5$ y 1,0 .

Se obtuvo la curva de reacción de la planta para los diferentes valores de y se identificó un modelo de primer orden más tiempo muerto en cada caso utilizando el método de dos puntos de Ho et al, 1995. Los parámetros de los modelos obtenidos se muestran en el Cuadro 7. A partir de los modelos identificados se calcularon los parámetros para controladores PI y PID-Ideal y se evaluaron los índices de desempeño mediante simulación con el programa VisSim para un intervalo de 0 a $30 \mathrm{~s}$ con un paso de integración de 0,001 s y el método de integración de RungeKutta de $4^{\circ}$ orden.

Los valores de los índices integrales IAE e ITAE obtenidos, se listan en el Cuadro 8 para los sistemas de control operando como regulador y en el Cuadro 9 para la operación como servomecanismo.

Para los controladores PI operando como regulador, se puede apreciar en el Cuadro 8 que el método de sintonización de Arrieta muestra ventaja sobre el de López para los valores altos de tanto para el índice IAE como el ITAE y que esta diferencia sólo es significativa para $=1,0$.
Para los controladores PID operando como regulador se puede apreciar en el Cuadro 8 que las diferencias entre los valores de los índices IAE e ITAE obtenidos con los diferentes métodos no son significativas, mostrando más bien una pequeña ventaja el método de López sobre el nuevo procedimiento para el caso de $=1,0$, mientras que éste supera al de Kaya y Sheib en todos los casos menos uno.

Para los controladores PI operando como servomecanismo se puede apreciar en el Cuadro 9 que el nuevo método muestra ventaja en los valores de los índices IAE e ITAE sobre los de Rovira en todos los casos, aunque esta no es significativa.

Para los controladores PID operando como servomecanismo se puede apreciar en el Cuadro 9 que en el método de Kaya y Sheib supera al de Rovira en los valores de los índices IAE e ITAE en todos los casos excepto en uno y que el nuevo procedimiento supera al de Rovira en todos los casos y al de Kaya y Sheib excepto en dos.

Los resultados anteriores concuerdan con los obtenidos en las pruebas comparativas realizadas con las plantas de primer orden más tiempo muerto empleadas en la obtención de los parámetros óptimos.

Cuadro 3. Reguladores PI - Valores de los índices de desempeño integral

$\begin{array}{cccccccc}\boldsymbol{t}_{\boldsymbol{m}} / & \text { Método } & \text { IAE } & \text { ITAE } & \boldsymbol{t}_{\boldsymbol{m}} / & \text { Método } & \text { IAE } & \text { ITAE } \\ & \text { López (IAE) } & 0,04 & - & 0,8 & \text { López (IAE) } & 1,16 & - \\ 0,1 & \text { Arrieta (IAE) } & 0,04 & - & & \text { Arrieta (IAE) } & 1,16 & - \\ & \text { López (ITAE) } & - & 0,06 & & \text { López (ITAE) } & - & 4,17 \\ & \text { Arrieta (ITAE) } & - & 0,06 & & \text { Arrieta (ITAE) } & - & 4,05 \\ & & & & & & & \\ & & & & & & \\ & \text { López (IAE) } & 0,45 & - & 1,2 & \text { López (IAE) } & 2,28 & - \\ & \text { Arrieta (IAE) } & 0,44 & - & & \text { Arrieta (IAE) } & 1,90 & - \\ & \text { López (ITAE) } & - & 1,11 & & \text { López (ITAE) } & - & 11,64 \\ & \text { Arrieta (ITAE) } & - & 1,07 & & \text { Arrieta (ITAE) } & - & 8,36\end{array}$


Cuadro 4. Servomecanismos PI - Valores de los índices de desempeño integral.

\begin{tabular}{|c|c|c|c|c|c|c|c|}
\hline$t_{m} /$ & Método & IAE & ITAE & $t_{m} /$ & Método & IAE & ITAE \\
\hline \multirow{4}{*}{0,1} & Rovira (IAE) & 0,21 & - & 0,8 & Rovira (IAE) & 1,56 & - \\
\hline & Arrieta (IAE) & 0,21 & - & & Arrieta (IAE) & 1,56 & - \\
\hline & Rovira (ITAE) & - & 0,25 & & Rovira (ITAE) & - & 3,23 \\
\hline & Arrieta (ITAE) & - & 0,24 & & Arrieta (ITAE) & - & 3,19 \\
\hline \multirow{4}{*}{0,4} & Rovira (IAE) & 0,83 & - & 1,2 & Rovira (IAE) & 2,43 & - \\
\hline & Arrieta (IAE) & 0,83 & - & & Arrieta (IAE) & 2,25 & - \\
\hline & Rovira (ITAE) & - & 1,29 & & Rovira (ITAE) & - & 5,83 \\
\hline & Arrieta (ITAE) & - & 1,29 & & Arrieta (ITAE) & - & 5,74 \\
\hline
\end{tabular}

Cuadro 5. Reguladores PID - Valores de los índices de desempeño integral.

\begin{tabular}{|c|c|c|c|c|c|c|c|}
\hline$t_{m} /$ & Método & IAE & ITAE & $t_{m} /$ & Método & IAE & ITAE \\
\hline \multirow{6}{*}{0,1} & López (IAE) & 0,02 & - & 0,8 & López (IAE) & 0,74 & - \\
\hline & Kaya (IAE) & 0,02 & - & & Kaya (IAE) & 0,81 & - \\
\hline & Arrieta (IAE) & 0,02 & - & & Arrieta (IAE) & 0,69 & - \\
\hline & López (ITAE) & - & 0,03 & & López (ITAE) & - & 2,09 \\
\hline & Kaya (ITAE) & - & 0,03 & & Kaya (ITAE) & - & 2,37 \\
\hline & Arrieta (ITAE) & - & 0,03 & & Arrieta (ITAE) & - & 2,06 \\
\hline \multirow{6}{*}{0,4} & López (IAE) & 0,24 & - & 1,2 & López (IAE) & 1,41 & - \\
\hline & Kaya (IAE) & 0,24 & - & & Kaya (IAE) & 1,62 & - \\
\hline & Arrieta (IAE) & 0,23 & - & & Arrieta (IAE) & 1,22 & - \\
\hline & López (ITAE) & - & 0,50 & & López (ITAE) & - & 5,09 \\
\hline & Kaya (ITAE) & - & 0,48 & & Kaya (ITAE) & - & 6,66 \\
\hline & Arrieta (ITAE) & - & 0,48 & & Arrieta (ITAE) & - & 4,69 \\
\hline
\end{tabular}

Cuadro 6. Servomecanismos PID - Valores de los índices de desempeño integral.

\begin{tabular}{|c|c|c|c|c|c|c|c|}
\hline$t_{m} /$ & Método & IAE & ITAE & $t_{m^{\prime}}$ & Método & IAE & ITAE \\
\hline \multirow{6}{*}{0,1} & Rovira (IAE) & 0,18 & - & 0,8 & Rovira (IAE) & 1,21 & - \\
\hline & Kaya (IAE) & 0,14 & - & & Kaya (IAE) & 1,15 & - \\
\hline & Arrieta (IAE) & 0,14 & - & & Arrieta (IAE) & 1,11 & - \\
\hline & Rovira (ITAE) & - & 0,30 & & Rovira (ITAE) & - & 2,92 \\
\hline & Kaya (ITAE) & & 0,15 & & Kaya (ITAE) & - & 3,83 \\
\hline & Arrieta (ITAE) & - & 0,15 & & Arrieta (ITAE) & - & 1,86 \\
\hline \multirow{6}{*}{0,4} & Rovira (IAE) & 0,65 & - & 1,2 & Rovira (IAE) & 1,85 & - \\
\hline & Kaya (IAE) & 0,58 & - & & Kaya (IAE) & 1,70 & - \\
\hline & Arrieta (IAE) & 0,57 & - & & Arrieta (IAE) & 1,67 & - \\
\hline & Rovira (ITAE) & - & 1,22 & & Rovira (ITAE) & - & 5,68 \\
\hline & Kaya (ITAE) & - & 0,98 & & Kaya (ITAE) & - & 10,27 \\
\hline & Arrieta (ITAE) & - & 0,76 & & Arrieta (ITAE) & - & 3,24 \\
\hline
\end{tabular}


Cuadro 7. Parámetros de los modelos de la planta de prueba.

$\begin{array}{llccl} & \mathbf{k} \mathbf{p} & \mathbf{t}_{\mathbf{m}} & & t_{m} / \\ 0,1 & 1,0 & 0,126 & 0,9831 & 0,128 \\ 0,2 & 1,0 & 0,264 & 0,9933 & 0,266 \\ 0,5 & 1,0 & 0,790 & 1,1360 & 0,695\end{array}$

Cuadro 8. Reguladores - Valores de los índices de desempeño integral, planta de prueba

0,1

\begin{tabular}{lcccc} 
& \multicolumn{2}{c}{ PI } & \multicolumn{2}{c}{ PID } \\
Método & IAE & ITAE & IAE & ITAE \\
López (IAE) & 0,05 & - & 0,03 & - \\
Kaya (IAE) & - & - & 0,04 & - \\
Arrieta (IAE) & 0,06 & - & 0,03 & - \\
López (ITAE) & - & 0,08 & - & 0,05 \\
Kaya (ITAE) & - & - & - & 0,05 \\
Arrieta (ITAE) & - & 0,09 & - & 0,04
\end{tabular}

$\begin{array}{lcccc}\text { López (IAE) } & 0,18 & - & 0,12 & - \\ \text { Kaya (IAE) } & - & - & 0,13 & - \\ \text { Arrieta (IAE) } & 0,21 & - & 0,12 & - \\ \text { López (ITAE) } & - & 0,39 & - & 0,22 \\ \text { Kaya (ITAE) } & - & - & - & 0,23 \\ \text { Arrieta (ITAE) } & - & 0,45 & - & 0,22\end{array}$

\begin{tabular}{llcccc} 
& & \multicolumn{2}{c}{ PI } & \multicolumn{2}{c}{ PID } \\
\multicolumn{1}{c}{0,5 Método } & IAE & ITAE & IAE & ITAE \\
López (IAE) & 1,02 & - & 0,62 & - \\
Kaya (IAE) & - & - & 0,66 & - \\
Arrieta (IAE) & 1,01 & - & 0,67 & - \\
López (ITAE) & - & 3,72 & - & 2,07 \\
Kaya (ITAE) & - & - & - & 2,15 \\
Arrieta (ITAE) & - & 3,68 & - & 2,16 \\
& & & & & \\
López (IAE) & 3,71 & - & 2,04 & - \\
Kaya (IAE) & - & - & 2,50 & - \\
Arrieta (IAE) & 3,11 & - & 2,20 & - \\
López (ITAE) & - & 29,81 & - & 13,40 \\
Kaya (ITAE) & - & - & - & 16,40 \\
Arrieta (ITAE) & - & 23,66 & - & 14,40
\end{tabular}

Cuadro 9. Servomecanismos - Valores de los índices de desempeño integral, planta de prueba

\begin{tabular}{|c|c|c|c|c|c|c|c|c|c|c|c|}
\hline & \multirow[b]{2}{*}{ Método } & \multicolumn{2}{|c|}{ PI } & \multicolumn{2}{|c|}{ PID } & & & \multicolumn{2}{|c|}{ PI } & \multicolumn{2}{|c|}{ PID } \\
\hline & & IAE & ITAE & IAE & ITAE & & Método & IAE & ITAE & IAE & ITAE \\
\hline \multirow{6}{*}{0,1} & Rovira (IAE) & 0,25 & - & 0,21 & - & 0,5 & Rovira (IAE) & 1,50 & - & 1,17 & - \\
\hline & Kaya (IAE) & - & - & 0,19 & - & & Kaya (IAE) & - & - & 1,15 & - \\
\hline & Arrieta (IAE) & 0,25 & - & 0,19 & - & & Arrieta (IAE) & 1,51 & - & 1,10 & - \\
\hline & Rovira (ITAE) & - & 0,34 & - & 0,31 & & Rovira (ITAE) & - & 3,47 & - & 2,52 \\
\hline & Kaya (ITAE) & - & - & - & 0,21 & & Kaya (ITAE) & - & - & - & 1,53 \\
\hline & Arrieta (ITAE) & - & 0,30 & - & 0,22 & & Arrieta (ITAE) & - & 3,39 & - & 2,00 \\
\hline \multirow{6}{*}{0,2} & Rovira (IAE) & 0,52 & - & 0,39 & - & 1,0 & Rovira (IAE) & 4,15 & - & 3,23 & - \\
\hline & Kaya (IAE) & - & - & 0,40 & - & & Kaya (IAE) & - & - & 3,01 & - \\
\hline & Arrieta (IAE) & 0,52 & - & 0,38 & - & & Arrieta (IAE) & 4,00 & - & 2,95 & - \\
\hline & Rovira (ITAE) & - & 0,80 & - & 0,66 & & Rovira (ITAE) & - & 16,58 & - & 12,30 \\
\hline & Kaya (ITAE) & - & - & - & 0,43 & & Kaya (ITAE) & - & - & - & 10,20 \\
\hline & Arrieta (ITAE) & - & 0,76 & - & 0,49 & & Arrieta (ITAE) & - & 16,17 & - & 9,26 \\
\hline
\end{tabular}




\section{CONCLUSIONES}

Con base en los resultados obtenidos en las simulaciones efectuadas, se puede afirmar que las nuevas ecuaciones obtenidas por Arrieta para el cálculo de los parámetros óptimos de controladores PI y PID-Ideal, bajo los criterios de desempeño integrales IAE e ITAE, a partir de un modelo de primer orden más tiempo muerto para la planta, proporcionan valores de estos índices en general mejores que los obtenidos con el método de López, el de Rovira o el de Kaya y Sheib, y que esta ventaja es mayor a medida que la relación $t_{m}$ /aumenta. Debe tenerse en cuenta que las nuevas ecuaciones de sintonización son válidas para valores de la relación $t_{m}{ }^{\prime} \quad 1,2$, mientras que las de los otros métodos comparados sólo lo son hasta un valor de 1,0.

Dadas las ventajas demostradas por las nuevas ecuaciones (8) a (10) para el cálculo de los parámetros de controladores PI y PID-Ideal, éstas pueden ser utilizadas en sustitución de las de los otros métodos tradicionalmente empleados para esto.

\section{SIMBOLOGÍA}

$G p(s)$ Función de transferencia (modelo).

$G c(s)$ Función de transferencia (controlador).

kp Ganancia estática.

i Constante de tiempo

tm Tiempo muerto aparente.

$s \quad$ Variable compleja.

Kc Ganancia del controlador.

Ti Tiempo integral.

Td Tiempo derivativo.

$e(t) \quad$ Señal de error.

$r(t) \quad$ Valor deseado.

$y(t) \quad$ Variable controlada.

$z(t) \quad$ Perturbación.

\section{NOTAS}

1. Proporcional-Integral-Derivativo

\section{BIBLIOGRAFÍA}

Alfaro, V. M.,(1993) ¿Son todos los controladores PID iguales?. Ingeniería, 3(1), 11-19.

Arrieta, O. (2003, Enero). Comparación del desempeño de los métodos de sintonización de controladores PI y PID basados en criterios integrales. Facultad de Ingeniería, Escuela de Ingeniería Eléctrica. San José, Costa Rica.

Aström, K. J. \& Hägglund, T. (2000). Benchmark Systems for PID Control, IFAC Workshop on Digital Control: Past, Present and Future of PID Control, Terrasa, España.

Coleman, T., Branch, M. H., Grace, A. (1999). Optimization toolbox for use with MATLAB. User's Guide, Version 2, The Matworks, Inc.

Ho, W. K., Hang, C. C., Cao, L. S. (1995). Tuning of PID controllers based on gain and phase margin specifications. Automatica, 31(3), 497-502.

Kaya, A. \& Sheib, T. J. (1988) Tuning of PID controllers of different structures. Control Engineering, December, 62-65.

López, A. M., Miller, J. A., Smith, C. L., Murrill, P. W. (1997). Tuning controllers with errorintegral criteria. Instrumentation Technology, November.

Rovira, A., Murrill, P. W., Smith, C. L. (1969) Tuning controllers for setpoint changes. Instrumentation \& Control Systems, December, 67-69. 
Sung, S. W., O, J., Lee, I. B., Lee, J., Yy, S. H. (1996). Automatic tuning of PID controller using second-order plus time delay model, Journal of Chemical Engineering of Japan, 29(6), 990-999.

Visual Solutions, (1999) VisSim user's guide version 3, Westford, Ma., EUA, Visual Solutions.

Wills, D. M. (1962a). Tuning maps for threeMode controllers. Control Engineering, April, 104-108.

Wills, D. M. (1962b) A guide to controllers tuning. Control Engineering, August, 93-94.

Ziegler, J. B. \& Nichols N. B. (1942). Optimum settings for automatic controllers. ASME Transactions, 64, 759-768.

\section{SOBRE LOS AUTORES}

\section{Orlando Arrieta Orozco}

Profesor del Departamento de Automática, Escuela de Ingeniería Eléctrica, Universidad de Costa Rica.

Apartado postal 2-10, 2060 UCR, San José, Costa Rica.

Teléfono: 207-4472, Facsímil: 207-4139

Correo electrónico: oarrieta@eie.ucr.ac.cr

\section{Víctor M. Alfaro Ruiz}

Profesor Asociado, Departamento de Automática, Escuela de Ingeniería Eléctrica, Universidad de Costa Rica.

Apartado postal 2-10, 2060 UCR, San José, Costa Rica.

Teléfono: 207-4472, Facsímil: 207-4139

Correo electrónico: valfaro@eie.ucr.ac.cr 
